\title{
Assessing the impact of the regular use (4 weeks) of an alcohol-based hand sanitizer on TEWL and epidermal hydration
}

\author{
Avaliação do impacto do uso regular (4 semanas) de um gel desinfetante na PTEA e \\ hidratação epidérmica
}

\author{
Henrique Silva ${ }^{1,2}$, Liliana Tavares ${ }^{1}$, Rita Faria ${ }^{1}$, Stefânia Delsin ${ }^{3}$, and L Monteiro Rodrigues ${ }^{1,2}$ \\ ${ }^{1}$ CBIOS - Universidade Lusófona's Research Center for Biosciences and Health Technologies (UDE), Campo Grande, 376, \\ 1749-024, Lisboa, Portugal \\ ${ }^{2}$ Pharmacol. Sc Depart - Universidade de Lisboa, School of Pharmacy, Lisboa, Portugal \\ ${ }^{3}$ Universidade de São Paulo, Faculty of Pharmaceutical Sciences of Ribeirão Preto, Avenida do Café, s/n, Ribeirão Preto, \\ Brazil \\ Email: henrique.silva@ulusofona.pt
}

\begin{abstract}
The Stratum corneum is a principal protector against physical, chemical, biological aggression and water loss in human skin. The repeated use of alcohol-based hand sanitizers might result in dryness and aggression. This study aims to evaluate the impact of a four week-long regular use of an alcohol-based gel hand sanitizer on skin hydration on the epidermal 'barrier' function using a kinetic model to quantify TEWL desorption curves following a POST test. A group of 13 female (19.7 \pm 1.0 years old) washed one of their hands for 29 consecutive days with a commercially available ethanol-based gel sanitizer. TEWL, superficial hydration (SH) and deep hydration (DH) were measured on days $1,8,15,22$ and 29 , while a continuous TEWL recording following a 24 hour POST was made on days 1, 15 and 29 on a second skin area. No differences were found for the control hand throughout the study. For the test hand an increase of TEWL was found between days 1 and 8, as well as an increase of SH and $\mathrm{DH}$ between days 1 and 22. No significant differences were found for the kinetic parameters. These results suggest that the frequent use of the sanitizer gel might alter the skin barrier function.
\end{abstract}

Keywords: hand sanitizer, TEWL, epidermal hydration, dynamic assessment

\section{Resumo}

O estrato córneo é o principal protetor contra a agressão física, química, biológica e perda de água na pele humana. A lavagem repetida das mãos especialmente com desinfetantes à base de álcool pode resultar em secura e agressão. Este estudo tem como objetivo avaliar o impacto do uso regular de quatro semanas de duração de um gel desinfetante para as mãos à base de álcool sobre a hidratação da pele sobre a função de 'barreira' epidérmica usando um modelo cinético para quantificar as curvas de dessorção de PTEA na sequência de um teste POST. Um grupo de 13 mulheres (19,7 $\pm 1,0$ anos de idade) lavaram as mãos durante 29 dias consecutivos com um desinfetante gel à base de etanol disponível no mercado. A PTEA, hidratação superficial (HS) e hidratação profunda (HP) foram medidas nos dias 1, 8, 15, 22 e 29, enquanto a PTEA foi registada continuamente na sequência de um POST de 24h nos dias 1, 15 e 29 numa segunda zona da pele. Não foram encontradas diferenças para a mão de controlo ao longo do estudo. Para a mão teste foi encontrado um aumento de PTEA entre os dias 1 e 8, bem como um aumento de $\mathrm{SH}$ e DH entre os dias 1 e 22. Nenhuma diferença significativa foi encontrada com os parâmetros cinéticos. Estes resultados sugerem que o uso frequente do gel desinfetante para as mãos pode alterar a função de barreira da pele.

Palavras-chave: desinfetante de mãos, PTEA, hidratação epidérmica, avaliação dinâmica 


\section{Introduction}

The human stratum corneum (SC), is a major determinant of the so called 'barrier' function, related to its capacity to protect the body against physical, chemical and biological aggressions, as well as against the excessive loss of water ${ }^{[1]}$. Excessive cleansing, as it commonly happens with repeated hand washing, especially with the use of alcohol-based sanitizers, is a mandatory practice in healthcare settings and reportedly evokes complaints of skin dryness ${ }^{[2,3]}$, which might reduce compliance with the practice ${ }^{[4,5]}$. The skin 'barrier' function can be quantified by the transepidermal water loss (TEWL), the water lost from deeper layers, through the stratum corneum to the surface ${ }^{[6]}$. When physically or functionally impaired, the epidermis tends to lose a higher amount of water into the environment, thus increasing the transepidermal water loss. Healthy skin tends to possess lower TEWL values and therefore, a larger amount of water in the stratum corneum ${ }^{[7]}$. The sensitivity of this variable is increased when employing provocation tests, including the commonly used plastic occlusion stress test (POST) ${ }^{[8-10]}$. This study aims to evaluate the impact of a four week-long regular use of an alcohol-based gel hand sanitizer on in vivo skin hydration and the epidermal 'barrier' function, using a previously established kinetic modelling of TEWL desorption curves following a POST test.

\section{Materials and Methods}

For this study, 13 healthy female volunteers $(19.7 \pm 1.0$ years old) were recruited, phototypes I to V (Fitzpatrick classification). Volunteers were informed of the experimental protocol and gave written consent to participate in the study, which was developed in accordance with the Declaration of Helsinki principles and respective amendments ${ }^{[11]}$. One randomly chosen hand was washed with $2 \mathrm{~mL}$ of a commercially available ( $\mathrm{Gel}$ desinfectante de mãos, Holon Produtos) sanitizer gel containing $80 \%$ ethanol (v/v) twice a day for 29 days (test hand). The contralateral hand was not treated, serving as a control. Two areas of approximately $16.0 \mathrm{~cm}^{2}$ were marked on the volunteers' back hands. In one of these (area 1) a dynamic provocation test took place. Volunteers were instructed not to apply any drugs or cosmetics in the tested areas. Area 1 was defined between the first and second fingers, while the area 2 was defined between the third and fourth fingers, centered halfway between the projection of ulnar apophysis and

\section{Introdução}

O estrato córneo (EC) humano é um principal determinante da função da chamada "barreira", relacionada com a sua capacidade de proteger o organismo contra as agressões biológicas, físicas e químicas assim como contra a perda excessiva de água ${ }^{[1]}$. Limpeza excessiva, como comumente acontece com lavagens repetidas, especialmente com a utilização de desinfetantes à base de álcool, é uma prática obrigatória nos serviços de saúde e alegadamente provoca queixas de secura da pele ${ }^{[2,3]}$, o que pode reduzir a adesão ${ }^{[4,5]}$. A função de "barreira" da pele pode ser quantificada pela Perda Transepidérmica de Água (PTEA), a água perdida através do estrato córneo para a superfície a partir de camadas mais profundas ${ }^{[6]}$. Quando agredida fisica ou funcionalmente, a epiderme tende a perder uma quantidade mais elevada de água para o meio ambiente, aumentando, assim, a PTEA. Portanto, uma pele saudável tende a possuir valores de PTEA mais baixos e, portanto, uma grande quantidade de água no estrato córneo ${ }^{[7]}$. A sensibilidade desta variável é maior quando se usam testes de provocação, incluindo o teste de oclusão plástica (POST) comumente usado ${ }^{[8-10]}$. Este estudo tem como objetivo avaliar o impacto do uso regular de quatro semanas de duração de um gel desinfetante à base de álcool na hidratação cutânea e na função epidérmica 'barreira' in vivo, usando um modelo cinético já estabelecido de curvas de dessorção de PTEA na sequência de um teste POST.

\section{Materiais e métodos}

Para este estudo foram recrutadas 13 voluntárias saudáveis (19,7 $\pm 1,0$ anos de idade), com fototipos de I a V (classificação de Fitzpatrick). As voluntárias foram informadas sobre o protocolo experimental, e deram o seu consentimento para participar no estudo, que foi desenvolvido em conformidade com a Declaração dos princípios de Helsínquia e respectivas revisões ${ }^{[11]}$. Uma mão escolhida aleatoriamente foi lavada com $2 \mathrm{~mL}$ de uma solução de desinfetante em gel contendo etanol $80 \%(\mathrm{v} / \mathrm{v})$ disponível no mercado (Gel desinfectante de Mãos, Holon Produtos) duas vezes por dia durante 29 dias (mão teste), servindo a mão contralateral como controlo. Duas áreas de aproximadamente $16,0 \mathrm{~cm}^{2}$ foram marcados nas costas das mãos das voluntárias. Em uma destas (zona 1) fez-se um teste de provocação dinâmica. As voluntárias foram orientadas a não aplicar quaisquer medicamentos ou cosméticos nas zonas testadas. A zona 1 foi definida entre o primeiro e segundo dedos, enquanto que a zona 2 foi definida entre o 
the base of the proximal phalanges. Measured variables of the skin were the transepidermal water loss (TEWL), surface hydration (SH) and deep hydration (DH). The plastic occlusion stress test (POST) was applied on days 1,15 and 29 in area 1 . Twenty-four hours after the patch removal, TEWL was recorded every 10 seconds $(0.1 \mathrm{~Hz}$ frequency) for 30 minutes. Area 2 was evaluated on days 1, 8, 15, 22 and 29. The occlusive patch was built as previously published ${ }^{[12]}$ (Figure 1). Before measurements, volunteers acclimatized for 20 minutes in a room with controlled temperature $\left(22 \pm 1{ }^{\circ} \mathrm{C}\right)$ and humidity (40-60\%). TEWL, expressed in $\left(\mathrm{g} / \mathrm{m}^{2} / \mathrm{h}\right)$ was measured with a Tewameter TM300 (Courage + Khazaka Electronics $\mathrm{GmbH}$, Cologne, Germany); superficial epidermal hydration ( $\mathrm{SH}$ ) expressed in arbitrary units (AU), using the MoistureMeter-SC ${ }^{\circledR}$ device (Delfin Technologies Ltd, Kuopio, Finland); and deep epidermal hydration (DH) expressed in arbitrary units (AU), using the MoistureMeter- ${ }^{\circledR}$ device (Delfin Technologies, Finland). The TEWL desorption curves were fitted to a bi-compartment kinetic model previously described ${ }^{[13-15]}$, using MatLab software (MATLAB and Statistics Toolbox Release 2011, The MathWorks, Inc., Natick, Massachusetts, USA), from which two kinetical parameters were extracted: $t_{1 / 2}$ evap (half-life of evaporation) and DWM (dynamic water mass). The evolution of the absolute TEWL values and of curve-derived kinetic parameters were determined in each hand and between control and test hands by the Wilcoxon signed-rank test (IBM SPSS Statistics for Windows, 20.0 version, Armonk, NY: IBM Corp). A 95\% confidence interval was adopted. terceiro e quarto dedos, centrada a meio caminho entre a projeção da apófise ulnar e a base das falanges proximais. As variáveis medidas da pele foram a Perda Transepidérmica de Água (PTEA), a hidratação superficial (HS) e a hidratação profunda (HP). O teste de oclusão plástica (POST) foi aplicado nos dias 1, 15 e 29 na zona 1. 24 horas após a remoção do penso a PTEA foi medida a cada 10 segundos (frequência de $0,1 \mathrm{~Hz}$ ), durante 30 min. A zona 2 foi avaliada nos dias 1, 8, 15, 22 e 29 . O penso oclusivo foi construído como previamente publicado ${ }^{[12]}$ (figura 1). Antes das medições, as voluntárias aclimatizaram durante 20 minutos numa sala com temperatura $\left(22 \pm 1^{\circ} \mathrm{C}\right)$ e humidade $(40-60 \%)$ controladas. A PTEA, expressa em $\left(\mathrm{g} / \mathrm{m}^{2} / \mathrm{h}\right)$ foi medida com um aparelho Tewameter TM300 (Courage + Khazaka Electronics $\mathrm{GmbH}$, Colónia, Alemanha); hidratação epidérmica superficial (HS) expressa em unidades arbitrárias (UA), utilizando o dispositivo MoistureMeter-SC $\AA$ (Delfin Technologies Ltd, Kuopio, Finlândia) e hidratação epidérmica profunda (HP), expressa em unidades arbitrárias (UA), utilizando o dispositivo MoistureMeter-D ${ }^{\circledR}$ (Delfin Technologies, Finlândia). As curvas de dessorção da PTEA foram ajustadas a um modelo cinético bi-compartimental já descrito ${ }^{[13-15]}$, utilizando o software MatLab (Toolbox MATLAB e Estatística Versão 2011, The MathWorks, Inc., Natick, Massachusetts, EUA), de onde foram extraídos dois parâmetros cinéticos: $t_{1 / 2}$ evap (tempo de meia-vida de evaporação) e DWM (massa de água dinâmica). A evolução dos valores absolutos da PTEA e dos parâmetros cinéticos derivados das curvas foi comparada para ambas as mãos e entre as mãos controlo e teste pelo teste teste não paramétrico de Wilcoxon para amostras emparelhadas $(\mathrm{p}<0.05)$. Foi adotado um intervalo de confiança de $95 \%$.

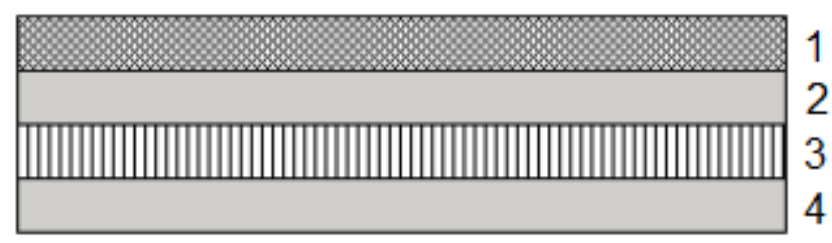

Figure 1/ Figura 1. Scheme of the occlusive patch used consisting of four layers, from top to bottom: 1 - Trumed Plaster; 2 - Parafilm; 3 - household plastic Cling Film; 4 Parafilm.

Esquema do penso oclusivo utilizado, consistindo de quatro camadas, de cima para baixo:

1 - penso Trumed; 2 - ParafilmTM; 3 - papel aderente; 4 - ParafilmTM. 


\section{Results and Discussion}

As shown in Table 1, TEWL increased progressively in the control hand from $t_{0}$ to $t_{2}$, and then diminished to $t_{3}$ and $t_{4}$. None of these changes was found to be statistically significant. On the test hand, however, TEWL increased significantly from $t_{0}$ to $t$, and then recovered progressively until $\mathrm{t}_{4}$. Superficial and deep hydration of the control hand did not change significantly throughout the protocol. On the test hand, higher $\mathrm{SH}$ and $\mathrm{DH}$ values were recorded from $\mathrm{t}_{1}$ throughout $t_{4}$, although statistical significance could only be found between $t_{0}$ and $t_{3}$. When comparing both hands, a significant increase of TEWL was found between $t_{4}$ and $t_{0}$. Regarding SH, significant differences were found between $t_{3}$ and $t_{0}$ and $t_{4}$ and $t_{0}$ and, for $D H$, between $t_{3}$ and $t_{0}$. This suggests that, in the present experimental conditions, the hand sanitizer affected the epidermal barrier function, as previously reported ${ }^{[12]}$, accepting that the increase of $\mathrm{SH}$ and DH could be attributed to the humectants included in the formulation ${ }^{[15]}$. Looking further to these TEWL desorption curves (Table 3 ), both $t_{1 / 2 \text { evap }}$ and DWM increased in both hands between $t_{0}$ and $t_{2}$ and between $t_{2}$ and $t_{4}$, but without statistically significance in any of these time intervals. Furthermore, no significant differences were found for these kinetic parameters when comparing the control with the test hand. In a recently published paper ${ }^{[12]}$, we presented results of a group of 13 volunteers that applied the very same sanitizer during 15 days, $4 \mathrm{~mL} /$ day, and significant differences on $t_{1 / 2 \text { evap }}$ were detected as soon as day 8 of application regarding day 1 . These results seem to indicate that ethanol-induced epidermal changes are dose-dependent, meaning that higher usage of this sanitizer might have more obvious, faster impact on the skin water dynamics.

\section{Resultados e discussão}

Como mostrado na tabela 1 a PTEA aumentou progressivamente de $t_{0}$ para $t_{2}$, e depois diminui para $t_{3}$ e $t_{4}$ na mão controlo. Nenhuma destas alterações se verificou ser estatisticamente significativa. Na mão teste, no entanto, a PTEA aumentou significativamente $t_{0}$ para $t_{1}$, e em seguida recuperou progressivamente até $t$. A hidratação superficial e profunda da mão de controlo nẩo se alterou significativamente ao longo do protocolo. Na mão teste, foram encontrados valores de HS e HP mais elevados a partir de $t_{1}$ até

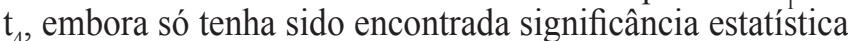
entre $t_{0}$ e $t_{3}$. Ao comparar as duas mãos, foi encontrado um aumento significativo de PTEA entre $t_{0}$ e $t_{4}$. Em relação à HS foram encontradas diferenças significativas entre $t_{0}$ e $t_{3}$ e entre $t_{0}$ e $t_{4}$ e para a HP entre $t_{0}$ e $t_{3}$. Isto sugere que, nas presentes condições experimentais, o desinfetante para as mãos afetou a função de barreira epidérmica, conforme relatado anteriormente ${ }^{[12]}$, aceitando que o aumento de HS e HP possa ser atribuído aos humectantes incluídos na formulação ${ }^{[15]}$. Olhando ainda para estas curvas de dessorção de PTEA (tabela 3), tanto $t_{1 / 2}$ evap como DWM aumentou em ambas as mãos entre $t_{0}$ e $t_{2}$, e entre $t_{2}$ e $t_{4}$, mas sem diferença estatística significativa em qualquer um desses intervalos de tempo. Além disso, não foram encontradas diferenças significativas para esses parâmetros cinéticos quando se compara a mão controlo com a mão teste. Num artigo publicado recentemente ${ }^{[12]}$, apresentámos resultados de um grupo de 13 voluntários que aplicou o mesmo desinfetatne durante 15 dias, $4 \mathrm{~mL} /$ dia e foram detetadas diferenças significativas no $_{1 / 2}$ evap logo ao dia 8 de aplicação em relação ao dia 1. Estes resultados parecem indicar que as alterações epidérmicas induzidas por etanol são dependentes da dose, o que significa que uma maior utilização deste desinfetante pode ter um impacto mais óbvio e mais rápido sobre a dinâmica da água epidérmica.

Table 1 / Tabela 1. Results (mean \pm standard deviation) obtained for the TEWL, SH and DH in the three measurement days ( $\mathrm{t} 0$ - day $1, \mathrm{t} 1$ - day $8, \mathrm{t} 2$ - day $15, \mathrm{t} 3$ - day $22, \mathrm{t} 4$ - day 29). Statistical comparison $(\mathrm{p}<0.05)$

for each hand between each measurement moment, for all volunteers $(n=13) . *$ - statistically significant.

Resultados (media \pm desvio-padrão) obtido para a PTEA, HS e HP nos três dias de medição (t0 - dia 1 , t1 dia 8, t2 - dia 15, t3 - dia 22, t4 - dia 29). Comparação estatística $(\mathrm{p}<0.05)$ para cada mão entre momentos de medição, para todos os voluntários $(\mathrm{n}=13) .{ }^{*}$ - estatisticamente significativo

\begin{tabular}{|c|c|c|c|c|c|c|c|c|c|c|c|}
\hline \multirow{3}{*}{\multicolumn{2}{|c|}{$\begin{array}{l}\text { Variable / } \\
\text { Variável }\end{array}$}} & \multirow{4}{*}{$\begin{array}{c}\begin{array}{c}\text { Control } \\
\text { hand / Mão } \\
\text { controlo }\end{array} \\
10.9 \pm 2.9 \\
\end{array}$} & \multirow{4}{*}{$\begin{array}{c}\text { Test hand / } \\
\text { Mão teste }\end{array}$} & \multicolumn{8}{|c|}{$p$-value / valor $p$} \\
\hline & & & & \multicolumn{4}{|c|}{ Control hand / Mão controlo } & \multicolumn{4}{|c|}{ Test hand / Mão teste } \\
\hline & & & & $t_{0} v t_{1}$ & $t_{0} v t_{2}$ & $t_{0} v s t_{3}$ & $t_{0} v t_{4}$ & $t_{0} v s t_{1}$ & $t_{0} v s t_{2}$ & $t_{0} v s t_{3}$ & $t_{0} v_{s} t_{4}$ \\
\hline \multirow{5}{*}{$\begin{array}{c}\text { TEWL / } \\
\text { PTEA } \\
\left(\mathrm{g} / \mathrm{h} / \mathrm{m}^{2}\right)\end{array}$} & $t_{0}$ & & & \multirow{5}{*}{0.286} & \multirow{5}{*}{0.209} & \multirow{5}{*}{0.754} & \multirow{5}{*}{0.239} & \multirow{5}{*}{$0.019^{*}$} & \multirow{5}{*}{0.055} & \multirow{5}{*}{0.552} & \multirow{5}{*}{0.600} \\
\hline & $t_{1}$ & $11.9 \pm 3.0$ & $12.5 \pm 3.6$ & & & & & & & & \\
\hline & $t_{2}$ & $12.7 \pm 3.0$ & $12.0 \pm 3.0$ & & & & & & & & \\
\hline & $t_{3}$ & $10.7 \pm 1.5$ & $11.0 \pm 3.6$ & & & & & & & & \\
\hline & $t_{4}$ & $9.6 \pm 3.4$ & $10.5 \pm 3.3$ & & & & & & & & \\
\hline \multirow{5}{*}{$\begin{array}{c}\mathrm{SH} / \mathrm{HS} \\
(\mathrm{AU})\end{array}$} & $\mathrm{t}_{0}$ & $26.5 \pm 9.1$ & $28.6 \pm 15.8$ & \multirow{5}{*}{0.695} & \multirow{5}{*}{0.092} & \multirow{5}{*}{0.388} & \multirow{5}{*}{0.367} & \multirow{5}{*}{0.087} & \multirow{5}{*}{0.485} & \multirow{5}{*}{$0.023^{*}$} & \multirow{5}{*}{0.124} \\
\hline & $t_{1}$ & $24.1 \pm 5.3$ & $29.0 \pm 6.7$ & & & & & & & & \\
\hline & $t_{2}$ & $23.1 \pm 4.6$ & $29.0 \pm 7.9$ & & & & & & & & \\
\hline & $t_{3}$ & $24.8 \pm 6.0$ & $34.1 \pm 7.1$ & & & & & & & & \\
\hline & $t_{4}$ & $24.4 \pm 4.6$ & $32.8 \pm 9.8$ & & & & & & & & \\
\hline \multirow{3}{*}{$\begin{array}{c}\mathrm{DH} / \mathrm{HP} \\
(\mathrm{AU})\end{array}$} & $t_{0}$ & $34.6 \pm 3.6$ & $33.8 \pm 3.9$ & \multirow{3}{*}{0.239} & \multirow{3}{*}{0.239} & \multirow{3}{*}{0.289} & \multirow{3}{*}{0.583} & \multirow{3}{*}{0.075} & \multirow{3}{*}{0.753} & \multirow{3}{*}{$0.003^{*}$} & \multirow{3}{*}{0.695} \\
\hline & $t_{1}$ & $35.2 \pm 4.2$ & $36.4 \pm 2.9$ & & & & & & & & \\
\hline & $\mathrm{t}_{2}$ & $34.8 \pm 3.3$ & $34.8 \pm 3.7$ & & & & & & & & \\
\hline
\end{tabular}


Table 2/ Tabela 2. Statistical comparison $(\mathrm{p}<0.05)$ of the variables' differences $(\Delta 1=\mathrm{t} 1-\mathrm{t} 0$; $\Delta 2=\mathrm{t} 2-\mathrm{t} 0 ; \Delta 3=\mathrm{t} 3-\mathrm{t} 0 ; \Delta 4=\mathrm{t} 4-\mathrm{t} 0)$ between control $(\mathrm{C})$ and test $(\mathrm{T})$ hands of all volunteers $(\mathrm{n}=13))^{*}$ - statistically significant.

Comparação estatística $(\mathrm{p}<0.05)$ das diferenças das variáveis $(\Delta 1=\mathrm{t} 1-\mathrm{t} 0 ; \Delta 2=\mathrm{t} 2-\mathrm{t} 0 ; \Delta 3=$ t3 - t0; $\Delta 4=\mathrm{t} 4-\mathrm{t} 0)$ entre as mãos controlo $(\mathrm{C})$ e teste $(\mathrm{T})$ para todos os voluntários $(\mathrm{n}=13)$.

* - estatisticamente significativo.

\begin{tabular}{|c|c|c|c|c|}
\hline \multirow{2}{*}{$\begin{array}{l}\text { Variable } \\
\text { Variável }\end{array}$} & \multicolumn{4}{|c|}{$p$-value / valor $p$} \\
\hline & $\Delta_{1} C$ vs $\Delta_{1} T$ & $\Delta_{2} C$ vs $\Delta_{2} T$ & $\Delta_{3} C$ vs $\Delta_{3} T$ & $\Delta_{4} C$ vs $\Delta_{4} T$ \\
\hline $\begin{array}{l}\text { TEWL / PTEA } \\
\left(\mathrm{g} / \mathrm{h} / \mathrm{m}^{2}\right)\end{array}$ & 0.315 & 0.570 & 0.191 & $0.023^{*}$ \\
\hline SH / HS (AU) & $0.023^{*}$ & 0.064 & $0.011^{*}$ & $0.017^{*}$ \\
\hline $\mathrm{DH} / \mathrm{HP}(\mathrm{AU})$ & 0.394 & 0.955 & $0.041^{*}$ & 0.221 \\
\hline
\end{tabular}

Table 3/ Tabela 3. Results (mean \pm standard deviation) obtained for the parameters $t 1 / 2$ evap and DWM in the three recording moments ( $\mathrm{t} 0$ - day 1 ; $\mathrm{t} 2$ - day 15; $\mathrm{t} 4$ - day 29). Statistical comparison $(\mathrm{p}<0.05)$ for each hand between $(\mathrm{C}$ - control, $\mathrm{T}$ - test) the two recording moments and for the difference between the values of the variables between the two hands $(\Delta 2=\mathrm{t} 2-\mathrm{t} 0 ; \Delta 4=\mathrm{t} 4-\mathrm{t} 0)$ of all volunteers $(\mathrm{n}=13) . *$ - statistically significant.

Resultados (media \pm desvio-padrão) obtidos para os parâmetros t1/2 evap e DWM nos três momentos de medição (t0 - dia 1; t2 - dia 15; t4 - dia 29). Comparação estatística $(\mathrm{p}<0.05)$ para cada mão $(\mathrm{C}$ - controlo, $\mathrm{T}$ - teste) entre momentos de medição e para a diferença entre os valores das variáveis entre as duas mãos $(\Delta 2=$ $\mathrm{t} 2-\mathrm{t} 0 ; \Delta 4=\mathrm{t} 4-\mathrm{t} 0)$ of all volunteers $(\mathrm{n}=13) . *$ - estatisticamente significativo.

\begin{tabular}{|c|c|c|c|c|c|c|c|c|c|}
\hline \multirow{3}{*}{\multicolumn{2}{|c|}{$\begin{array}{l}\text { Parameter I } \\
\text { Parâmetro }\end{array}$}} & \multirow{3}{*}{$\begin{array}{l}\text { Control hand / } \\
\text { Mão controlo }\end{array}$} & \multirow{3}{*}{$\begin{array}{c}\text { Test hand / Mão } \\
\text { teste }\end{array}$} & \multicolumn{6}{|c|}{$p$-value / valor $p$} \\
\hline & & & & \multicolumn{2}{|c|}{$\begin{array}{c}\text { Control hand / Mão } \\
\text { controlo }\end{array}$} & \multicolumn{2}{|c|}{$\begin{array}{c}\text { Test hand / Mão } \\
\text { teste }\end{array}$} & \multirow{2}{*}{$\begin{array}{c}\Delta_{2} C \text { vs } \\
\Delta_{2} T\end{array}$} & \multirow{2}{*}{$\begin{array}{c}\Delta_{4} C \text { vs } \\
\Delta_{4} \mathrm{~T}\end{array}$} \\
\hline & & & & $t_{0} v^{\prime} t_{2}$ & $t_{0} v s t_{4}$ & $t_{0} v s t_{2}$ & $t_{0} v s t_{4}$ & & \\
\hline \multirow{3}{*}{$\begin{array}{l}\mathbf{t} 1 / 2 \\
\text { evap } \\
\text { (min) }\end{array}$} & $t_{0}$ & $5.40 \pm 2.82$ & $5.58 \pm 1.93$ & \multirow{3}{*}{0.124} & \multirow{3}{*}{0.116} & \multirow{3}{*}{0.196} & \multirow{3}{*}{0.235} & \multirow{3}{*}{0.382} & \multirow{3}{*}{0.807} \\
\hline & $t_{2}$ & $7.21 \pm 4.31$ & $6.61 \pm 2.51$ & & & & & & \\
\hline & $t_{4}$ & $7.12 \pm 5.32$ & $7.38 \pm 3.36$ & & & & & & \\
\hline \multirow{3}{*}{$\begin{array}{l}\text { DWM } \\
\left(\mathrm{g} / \mathrm{m}^{2}\right)\end{array}$} & $t_{0}$ & $7107.62 \pm 2618.94$ & $7686.87 \pm 2172.72$ & \multirow{3}{*}{0.064} & \multirow{3}{*}{0.060} & \multirow{3}{*}{0.116} & \multirow{3}{*}{0.116} & \multirow{3}{*}{0.600} & \multirow{3}{*}{0.311} \\
\hline & $t_{2}$ & $8660.57 \pm 2062.78$ & $8734.05 \pm 1890.73$ & & & & & & \\
\hline & $\mathbf{t}_{4}$ & $8807.72 \pm 2534.29$ & $8609.48 \pm 2128.07$ & & & & & & \\
\hline
\end{tabular}

\section{Conclusions}

These results seem to confirm that there is still need to improve these formulations, especially when their use is regularly repeated as happens in specific environments such as healthcare spaces. It also demonstrates the usefulness of this kinetic model to detect subtle differences in epidermal water dynamics.

\section{Conclusões}

Estes resultados parecem confirmar que há ainda a necessidade de melhorar estas formulações, especialmente quando a sua utilização é repetida regularmente, como acontece em ambientes específicos (espaços de saúde). Demonstram também a utilidade deste modelo cinético para detectar diferenças subtis na dinâmica da água epidérmica. 


\section{Acknowledgements}

The authors would like to express their thanks to all volunteers.

\section{Conflict of Interests}

The authors declare that there are no financial and/or personal relationships that could be viewed as presenting a potential conflict of interests.

\section{Agradecimentos}

Os autores expressam os seus agradecimentos a todas as voluntárias.

\section{Conflito de Interesses}

Os autores declaram não existir qualquer relação pessoal ou financeira que possa ser entendida como representando um potencial conflito de interesses. 


\section{References/ Referências}

1. Rosado C, Ferreira J, Pinto PC, Rodrigues LM . Skin barrier function evaluation by bicompartmental analysis of tewl dynamical measurements: validation of new analytical conditions. Biomed Biopharm Res 2012; (9) 2: 183-189.

2. Larson EL, Early E, Cloonan P, Sugrue S, Parides M. An organizational climate intervention associated with increased handwashing and decreased nosocomial infections. Behav Med 2000; 26:14-22.

3. Boyce JM, Pittet D; Healthcare Infection Control Practices Advisory Committee, HICPAC/SHEA/APIC/IDSA Hand Hygiene Task Force Guideline for hand hygiene in health-care settings. Recommendations of the Healthcare Infection Control Practices Advisory Committee and the HICPAC/ SHEA/APIC/IDSA Hand Hygiene Task Force. MMWR Recomm Rep 2002; 51(RR16): $1-45$.

4. Löffler H, Kampf G, Schmermund D, Maibach HI. How irritant is alcohol? Br J Dermatol 2007; 157(1):74-81.

5. Houben E, De Paepe K, Rogiers V. Skin condition associated with intensive use of alcoholic gels for hand disinfection: a combination of biophysical and sensorial data Contact Dermatitis 2006; 54(5):261-7.
6. Lotte C, Rougier A, Wilson DR, Maibach HI. In vivo relationship between transepidermal water loss and percutaneous penetration of some organic compounds in man: effect of anatomic site. Arch Dermatol Res 1987; 279: 351-356.

7. Rosado C, Rodrigues LM. Assessment of dry skin using dynamic methods. J. Appl. Cosmetol 2006; 24: 139-157.

8. Berardesca E, Fideli D, Gabba P, Cespa M, Rabbiosi G, Maibach HI. Ranking of surfactant skin irritancy in vivo in man using the plastic occlusion stress test (POST). Contact Dermatitis 1990; 23 (1):1-5.

9. Pinto $\mathrm{P}$, Rosado $\mathrm{C}$, Parreirão $\mathrm{C}$, Rodrigues LM. Is there any barrier impairment in sensitive skin?: a quantitative analysis of sensitive skin by mathematical modeling of transepidermal water loss desorption curves. Skin Res Technol 2011; 17(2):181-5.

10. Rodrigues LM, Silva H., Rosado C, Regarding the quantification of the human skin barrier in Sensitive Skin Syndrome, Honari G, Andersen R, Maibach HI, eds. Sensitive Skin Syndrome, second edition, CRC Press: Boca Raton (in press)
11. World Medical Association. World Medical Association Declaration of Helsinki: ethical principles for medical research involving human volunteers. JAMA; 310(20):2191-4.

12. Silva H, Silva SA, Ferreira H, Rodrigues LM. Assessing the in vivo impact of a gel sanitizer on the epidermal "barrier" dynamics. Biomed Biopharm Res 2015; (12) 1: 69 77.

13. Rodrigues LM, Pinto PC, Galego N, Da Silva PA, Pereira LM. Transepidermal water loss kinetic modeling approach for the parameterization of skin water dynamics. Skin Res Technol 1999; 5:72-82.

14. Rosado C, Pinto P, Rodrigues LM. Modeling TEWL-desorption curves: a new practical approach for the quantitative in vivo assessment of skin barrier. Exp Dermatol 2005 14(5):386-90.

15. Dal'Belo SE, Gaspar LR, Maia Campos PM Moisturizing effect of cosmetic formulations containing Aloe vera extract in different concentrations assessed by skin bioengineering techniques. Skin Res Technol. 2006;12(4):241-6. 